\title{
Fusion of 3D B-spline surface patches reconstructed from image sequences
}

\author{
Roger Mohr ChangSheng Zhao Gautier Koscielny \\ LIFIA-INRIA \\ 46 Avenue Félix Viallet \\ 38031 Grenoble cedex France
}

\begin{abstract}
This paper considers the problem of merging a set of distinct three dimensional B-spline surface patches, which are reconstructed from observations of the motion of occluding contours in image sequences [6, 7]. We propose an original method of fusing these partially overlapping patches in order to obtain a whole surface. This approach is based on a triangular mesh and surface interpolation through regularized uniform bicubic B-spline surface patches. Experimental results are presented for both synthetic and real data.
\end{abstract}

\section{Introduction}

One of the aims of computer vision is to acquire robust surface descriptions from several images of a scene. The accurate descriptions can be used for manufacturing automation, terrain mapping, vehicle guidance, surveillance, virtual reality, etc. Much progress has been made recently in the field of non polyhedral objects surface reconstruction $[2,3,6]$.

From the perspective of classical differential geometry, there are three representations for a surface of an object: explicit, implicit, and parametric. In our work [6], we have used uniform bicubic B-spline surface patches to give a parametric representation of a non polyhedral object surface. Our aim is to reconstruct a $3 \mathrm{D}$ whole surface of the object from the observation of occluding contours together with the knowledge of the motion of the camera mounted on the robot. We have shown that the reconstruction of such a $3 \mathrm{D}$ surface is possible when the camera motion is known, with the exception of those fully concave parts where no view line can be tangential to the surface and the contour is singular [4].

But our method of surface reconstruction corresponds only to a single motion which allows us to reconstruct a part of the 3D surface of an object. Due to occlusion or constraints on the motion and some diffculties we encountered with the epipolar parameterisation [6] which are pointed out in the work of Giblin \& Weiss [4], several B-spline surface patches of a single object are then obtained and have to be fused. The fusion problem has been addressed in Computer-Aided Designs and Computer Graphics. Volume or surface models are used for the creation of forms. Surface models are widely used in the aeronautics, automobile, naval and other industries. They permit "thin plate" objects to be modeled realistically. Large complicated surfaces are generally modeled by a serie of discrete surface patches following the spline model. Two kinds of problems arise: the first one is creation of 3D surface pieces and positioning them in space, the second one is fusion of the distinct surface pieces to obtain a global surface. Here we consider surface patches fusion in the context of computer vision. However because the data are experimentally derived the surface patches may overlap or there may be gaps. Each patch is also independently parametrised. The fusion of those distinct B-spline surface patches can be achieved by returning to a discrete representation with a grid of 3D points sampled on the surface patches, net fusion then becomes a much simpler process.

This paper is composed of two parts: in the first one we will introduce in detail our algorithm for merging two distinct B-spline surface patches. In the second part, we present experimental results on synthetic and real data.

\section{Our approach}

The parametric representation of an object surface by using uniform bicubic B-spline surface patches is unable to deal directly with the problem of fusion. Therefore, we decide to use an intermediate discrete representation of the surface: a triangular mesh. In 
the following, we will introduce in detail an efficient algorithm of fusing two distinct B-spline surface patches. This process can be decomposed into the four following steps:

- triangular mesh of B-spline surface patches.

- common regions detection for the two distinct Bspline surface patches.

- surface interpolation of a set of 3D points.

- fusion of the two distinct B-spline surface patches.

\subsection{Triangular mesh}

With this parameterisation of B-spline surface patches, we can obtain a set of discrete $3 \mathrm{D}$ points. We also have geometric information at these points normal vectors and Gaussian and mean curvatures. Hence this discretisation of a surface can be represented by a graph. The nodes of the graph are given by the points sampled from B-spline surface patches. The arcs of the graph are presented by the neighbourhood relations among these points. We note that a node has at most eight neighbourhood relations. This graph allows us to produce a regular mesh. In our case, we built a triangular mesh selecting six neighbourhood nodes [7]. Because it is fast and it conserves the information of the principal neighbourhood relations.

From now on, we will define a new structure which represents a node of the graph and includes the necessary geometric and topological information. First this structure must have the following geometric information: coordinates of the point of the surface in 3D space, normal vector to this point, and the normal vector. Secondly, the structure must also contain the following topological information: list of the neighbourhood nodes, its position in the graph (border, inside).

\subsection{Common regions detection}

Now let us suppose that we have two meshed Bspline surface patches which overlap. From here, we can detect their common regions. The idea is to choose rules for verifying if a point belongs to the common regions of these two patches. The algorithm is the following: let $A$ and $B$ be two overlapping patches. We take a point $a \in A$, if it exists at least a point $b \in B$ such that

$$
\left\{\begin{array}{l}
|a-b| \leq \epsilon \\
<\vec{N}_{a}, \vec{N}_{b}>\leq \theta
\end{array}\right.
$$

where $\epsilon$ is the Euclidean distance chosen between two points by our experiments, $\vec{N}_{i}$ is the normal vector at the point $i,\langle\cdot, \cdot\rangle$ represents the angle between two normal vectors.

The first condition of (1) means that a point $a$ of the patch $A$ belongs to a common region if it exists a point of the patch $B$ which is sufficiently near the point $a$. The second condition of (1) allows us to eliminate the neighbourhood points which have different curvatures. In practice, these thresholds $\varepsilon$ and $\theta$ are obtained through experiment. When a common region is extracted, the points of the region border are stamped as border information and stored with the number of the region.

\subsection{Treatment of the common regions ex- tracted}

From the above paragraph, we can detect the common regions of two B-spline surface patches as a set of $3 \mathrm{D}$ points. The result is an only partially structured set of points as the mesh structure is partially destroyed and no connection is made between the two sets of points. In this paragraph, first we will propose a method for obtaining an optimal surface interpolating the data set. Secondly, this new surface will be reduced with respect to two initial B-spline surface patches.

For the surface interpolation of a set of $3 \mathrm{D}$ points, we need to find the mean direction of a set of points. What is this direction? Suppose that we have a set of points $\left(x_{k}, y_{k}, z_{k}\right)_{k=1, \ldots, p}$ in the old coordinate frame $O x y z=(O, \vec{i}, \vec{j}, \vec{k})$. the mean direction $\vec{d}=\left(d_{x}, d_{y}, d_{z}\right)^{T}$ of this set of points is defined by

$$
\left\{\begin{array}{l}
d_{x}=\sum_{k=1}^{p} \frac{x_{k}}{p} \\
d_{y}=\sum_{k=1}^{p} \frac{y k}{p} \\
d_{z}=\sum_{k=1}^{p} \frac{z_{k}}{p} .
\end{array}\right.
$$

From now on, we can define the new coordinate frame $O X Y Z=(O, \vec{I}, \vec{J}, \vec{K})$ such that the axis $O Z$ is parallel to the mean direction $\vec{d}$ (see Figure 1). Therefore, it remains only to interpolate the set of points in the new coordinate frame. In our case, we selected to use uniform bicubic B-spline surface patches for surface interpolation. For simplicity, uniform bicubic B-spline surface patches can be expressed by the following formula:

$$
F(x, y)=\sum_{i=-3}^{m-1} \sum_{j=-3}^{n-1} \alpha_{i j} B_{i}(x) B_{j}(y) .
$$




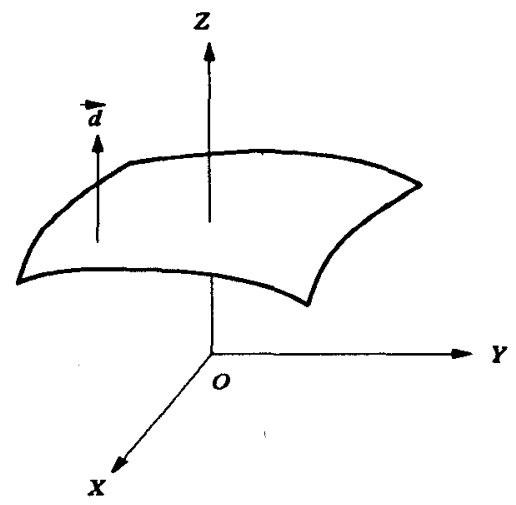

Figure 1: The mean direction $\vec{d}$ of a set of points is parallel to the axis $O Z$.

Therefore, this problem is equivalent to fitting the Bspline surface patches to the data points. More precisely, B-spline surface patches interpolation requires the estimation of $(m+3) \times(n+3)$ unknows $\alpha_{i j}$, $i=-3, \ldots, m-1, j=-3, \ldots, n-1$ in the formula (3).

Since the set of $3 D$ points are discretized from the B-spline patches, an input as the data set of the process of our surface interpolation is not very accurate. Usually, least squares method does not allow the form of our surface to be easily adjusted. We therefore introduce a regularization term on the surface $[1,7]$. The term allows us to minimize the total variation in the local surface orientation. This suggests that the term should measure some factor of the second-order derivatives of the surface. Finally, we use the following regularization term (4) [7]. The minimization function for our surface interpolation is then defined by

$\Theta=\sum_{k=1}^{p}\left[F\left(x_{k}, y_{k}\right)-z_{k}\right]^{2}+\tau \int_{I} \int_{J}\left(\frac{\partial^{6} F}{\partial^{3} x \partial^{3} y}\right)^{2} d x d y$

where the factor $\tau \in \mathbb{R}^{+}$is the tensor of B-spline surface patches.

The variational conditions of optimization express the fact that the first partial derivative of $\Theta$ relative to $\alpha_{i j}$ is zero:

$$
\frac{\partial \Theta}{\partial \alpha_{i j}}=0
$$

where $i=-3, \ldots, m-1, j=-3, \ldots, n-1$. We remark that $B_{i}$ is a piecewise polynomial function of degree 3 and $\frac{\partial^{3} B_{i}(x)}{\partial^{3} x} \frac{\partial^{3} B_{j}(y)}{\partial^{3} y}$ is then constant on every interval.

Obviously, the system (5) can be rewritten as the following linear system $M \cdot X=B$ where $M$ is a $(m+3)(n+3) \times(m+3)(n+3)$ symmetric matrix.
Finally, the above linear system can be solved by classical methods: Cholesky decomposition or singular value decomposition or LU decomposition [7]. Therefore, we can obtain B-spline surface patches interpolated from a set of $3 \mathrm{D}$ points.

Reconstruction of a unique mesh is then straightforward. If we compare this new surface with two initial B-spline surface patches, we note that there is a part which must be eliminated. The principle of the reduction of the new surface is very simple: we project the new surface towards the axis $O Z$, if a point is inside of the common region detected, then it will be kept, otherwise it will be rejected. Once this process is complete, we have to change all the coordinates of the points from the new coordinate frame to the old coordinate frame.

\subsection{Fusion of two different patches}

The problem is now to fuse the two close patches. A solution was proposed by the work of Turk et al. [5]. Our approach differs with previous work. First, the points of the new reduced surface border are given by border information. Secondly, we fuse each stamped point with the nearest point of the new reduced surface border which have the border information. The condition to be respected here is that the sum of the number of neighbourhood relations of the fused points must be inferior or equal to six. After these steps, we eliminate the points to be fused. The points that we can not eliminate are those inside the patches.

\section{Experimental results}

In this paragraph, we demonstrate our method on synthetic and real data.

First we present the results of fusing two distinct B-spline surface patches of a synthetic hyperboloid, obtained by two different motions of the camera.

Figure 2 shows two B-spline surface patch reconstructions of a hyperboloid when the view point of the camera is changed.

Figure 3 shows the superposition of the two Bspline surface patches from Figure 2.

Figure 4 shows the non overlapping regions of the two B-spline surface patches from Figure 2.

Figure 5 presents the result of the fusion of two B-spline surface patches.

We demonstrate also the fusion of two B-spline surface patches reconstructed of a carafe. 

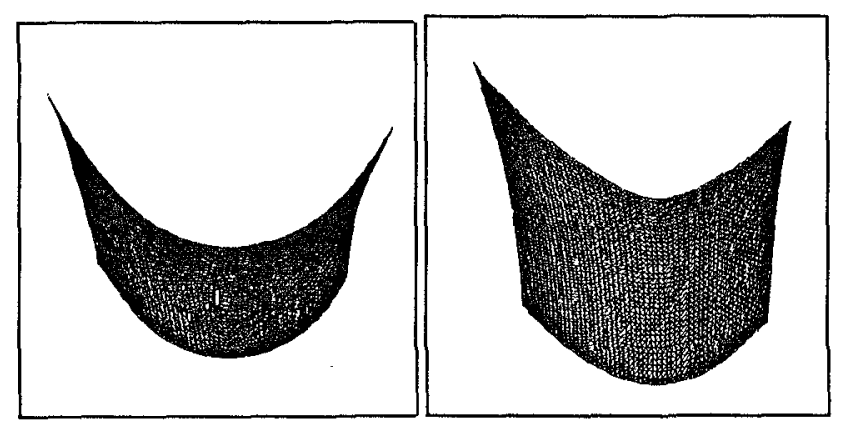

Figure 2: First patch (left) and second patch (right) of B-spline surface reconstructed of a hyperboloid.

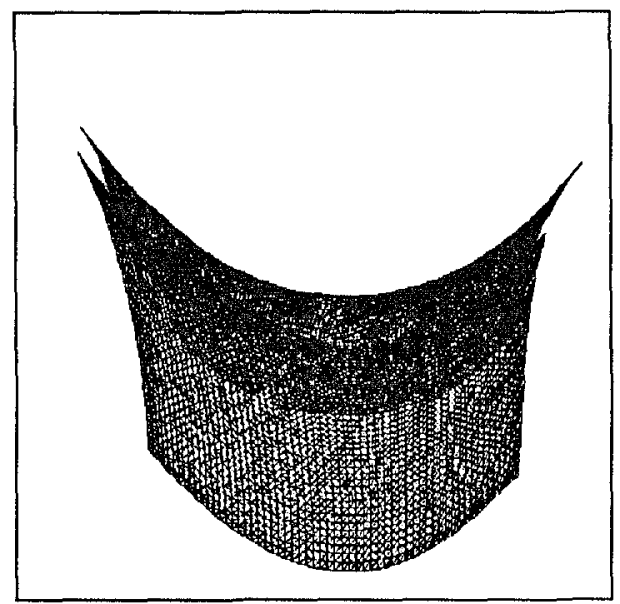

Figure 3: Superposition of two B-spline surface patches

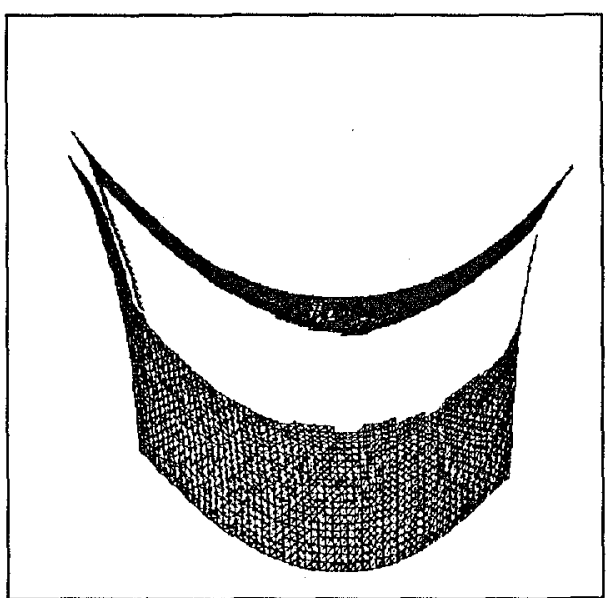

Figure 4: Extraction of the common regions detected of two B-spline surface patches

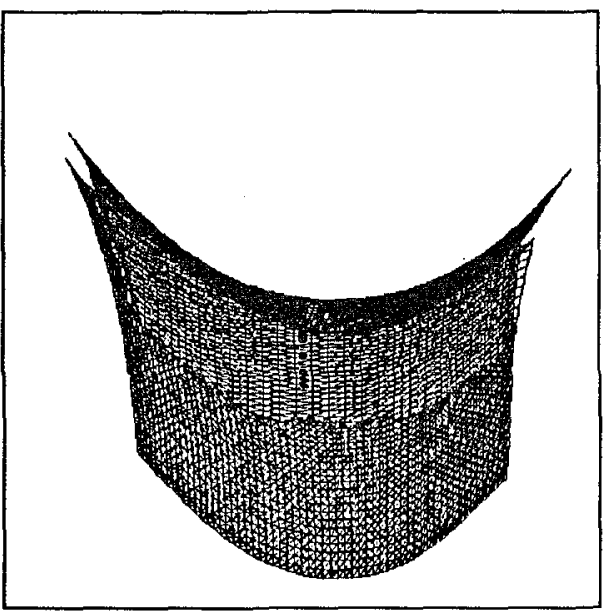

Figure 5: Fusion of two B-spline surface patches

Figure 6 presents the result of triangular mesh of two B-spline surface patches reconstructed from the carafe [7].
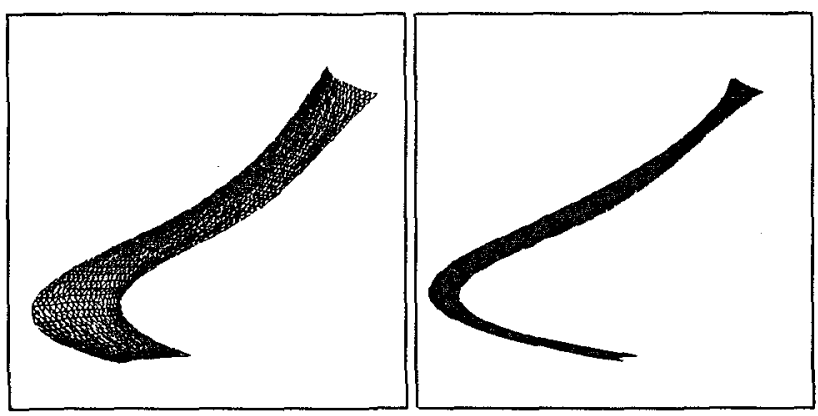

Figure 6: First patch (left) and second patch (right) of B-spline surface reconstructed from the carafe

Figure 7 shows the superposition of two B-spline surface patches reconstructed from the carafe.

In Figure 8, the remainder of the two B-spline surface patches is displayed, after extracting the common regions.

Figure 9 presents the result of the fusion of two B-spline surface patches.

We have the following remarks:

Remark 1: we remark that some points could not be extracted. Indeed, these points do not verify the selection rules given by the two inequalities (1). In practice, the thresholds of $\varepsilon$ and $\theta$ are chosen by our experiments.

Remark 2: we note in Figure 8 that the curvature changes strongly on the border of the reconstructed B-spline surface patches, and the quality of the fusion is altered. 


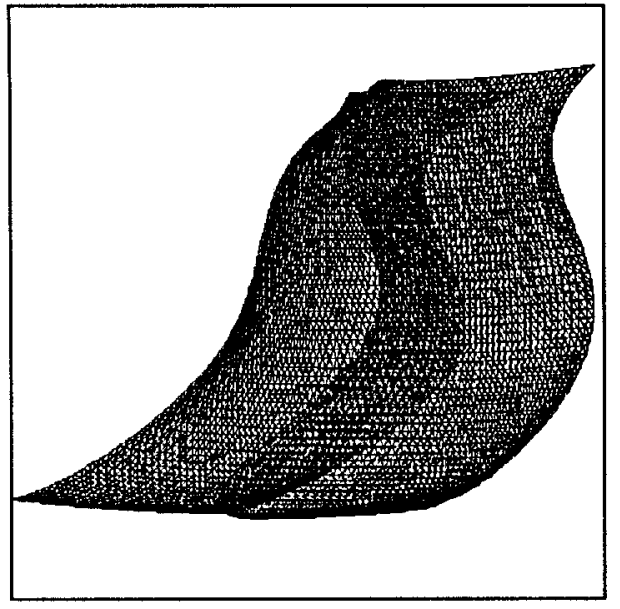

Figure 7: Superposition of two B-spline surface patches

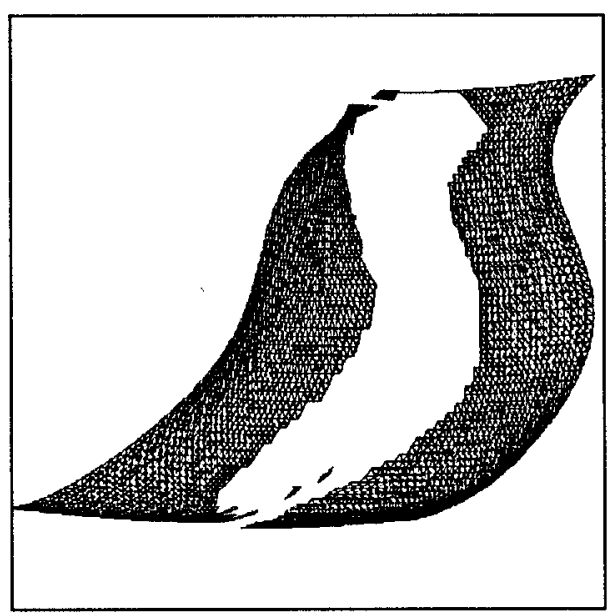

Figure 8: Extraction of the common regions detected of two B-spline surface patches

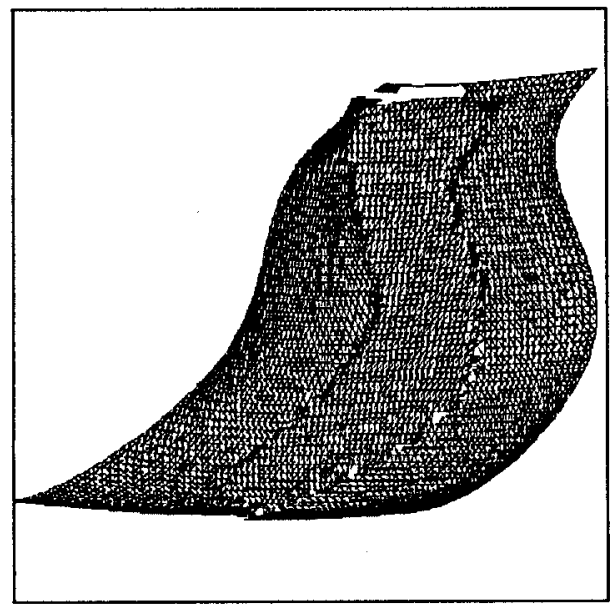

Figure 9: Fusion of two B-spline surface patches
Remark 3: we remark also the change of triangular mesh orientation between the initial B-spline surface patches and the cut regions.

\section{Perspective and Conclusions}

In this paper, a novel efficient method of fusing two B-spline surface patches has been demonstrated. The fusion process provided interesting results. In particular, the triangular mesh structure allows us easily to establish the neighbourhood relations amongst the points of a patch in order to deal with geometric and topological information. Our surface interpolation method of a set of $3 \mathrm{D}$ points through regularized uniform bicubic B-spline surface patches is very simple and efficient. It is very easy to apply this fusing method recursively to a set of different B-spline surface patches in order to obtain a complete meshed surface for an object. Future work will ameliorate the faults of fusing method currently implemented and consider the application of the reconstructed surface for object recognition, navigation and grasping tasks.

\section{References}

[1] P.J. Laurent, Courbes ouvertes ou fermées par B-splines régularisées, TR, RR 652-M-, IMAG, Grenoble, France, 1987.

[2] R. Cipolla and A. Blake, Surface Shape from the Deformation of Apparent Contours, IJCV, $9(2): 83-112,1992$.

[3] R. Vaillant and O.D. Faugeras, Using Extremal Boundaries for 3D Objet Modeling, PAMI, 14(2):157-173, 1992.

[4] P.J. Giblin and R. Weiss, Epiolar fields on surfaces, ECCV, Vol. 800, 14-23, 1994.

[5] G. Turk and M. Levoy, Zippered polygon meshs from range images, SIGGRAPH, to appear, 1994.

[6] C.S. Zhao and R. Mohr, Relative 3D regularized B-spline surface reconstruction through image sequences, ECCV, Vol. 801, 417-426, 1994.

[7] C.S. Zhao and R. Mohr, Global three dimensional surface reconstruction from occluding contours, $T R$, RT 116 IMAG-25 LIFIA, IMAG, Grenoble, France, 1994. 